\title{
Move to get moved: a search for methods, tools and knowledge to design for expressive and rich movement-based interaction
}

\author{
Caroline Hummels · Kees C. J. Overbeeke • \\ Sietske Klooster
}

Received: 11 December 2005/ Accepted: 2 September 2006/ Published online: 14 November 2006

(C) Springer-Verlag London Limited 2006

\begin{abstract}
The world is inherently meaningful for us, i.e. we perceive the world in terms of what we can do with it, and by physically interacting with it we access this meaning and express the meaning. We believe that this is the core reason and foundation for turning to movement-based interaction. 'Interaction creates meaning' does not only hold for users during interaction but also for designers when generating ideas and developing concepts. Therefore, we postulate that if one truly likes to design for movement-based interaction, one has to be or become an expert in movement, not just theoretically, by imagination or on paper, but by doing and experiencing while designing. In order to do so, we believe that designers need design tools, techniques, knowledge, awareness and skills that support their search for expressive, rich behaviour. Our search for
\end{abstract}

C. Hummels $(\bowtie)$

Faculty of Industrial Design Engineering,

Delft University of Technology, Landbergstraat 15,

2628 CE Delft, The Netherlands

e-mail: C.C.M.Hummels@io.tudelft.nl

URL: http://studiolab.io.tudelft.nl/hummels

K. C. J. Overbeeke · S. Klooster

Department of Industrial Design,

Eindhoven University of Technology, Den Dolech 2,

5612 AZ Eindhoven, The Netherlands

K. C. J. Overbeeke

e-mail: C.J.Overbeeke@tue.nl

S. Klooster

e-mail: sietske@designmovement.nl

S. Klooster

Design Movement, Berberisstraat 16,

1032 EL Amsterdam, The Netherlands

e-mail: sietske@designmovement.nl this support resulted in several methods, tools and knowledge that help designers exploring, visualising and reflecting on interactions. Our developed methods and tools such as the Design Movement approach with its choreography of interaction, gestural design tools, interactive installations and interactive tangible sketching, have not only supported and inspired designers to design for movement-based interaction, but also resulted in surprising, fresh designs in comparison with the limited scope of rather uniform and traditional electronic consumer products. This paper discusses the possibilities and limitations of our approach.

Keywords Movement-based interaction - Richness · Tangible interaction - Meaning · Tools · Methods . Gestures · Choreography of interaction · Installations · Emotions · Expressivity - Experience · Product design

\section{Introduction}

When looking at the juggler in Fig. 1, one can image how he is juggling. With little imagination, one can visualise the path of the balls in relation to the movements the juggler is making. However, if you have ever juggled yourself, or tried to do so, you know that imagining is not the same as experiencing. That is why jugglers need extensive training to become experts and do those amazing tricks with an almost impossible number of burning torches or weird objects.

We believe that the same holds for design. If one truly likes to design for movement-based interaction, one has to be an expert in movement, not just theoretically, by imagination or on paper, but by doing and experiencing while designing. 


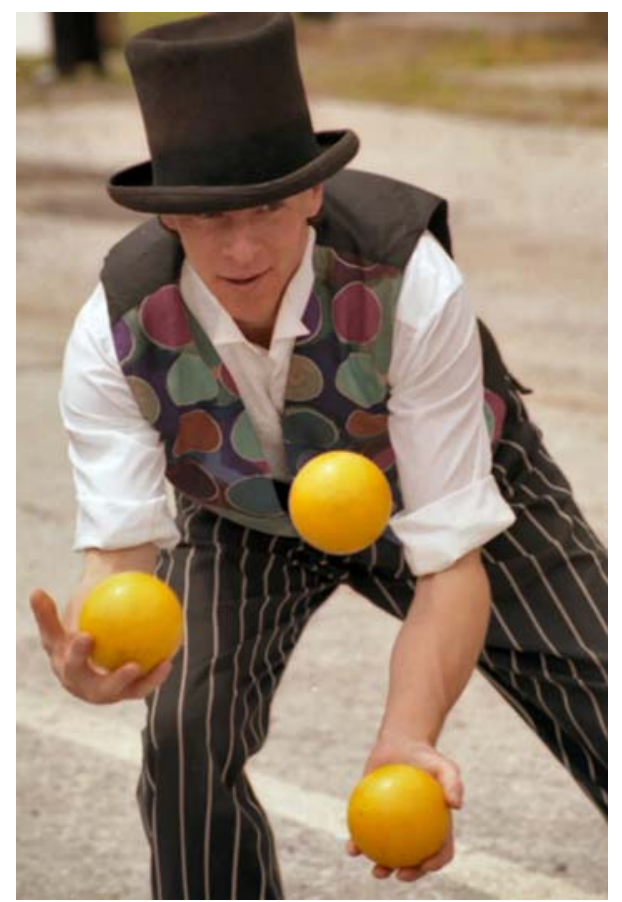

Fig. 1 Imagining how this juggler is handling the balls is not the same as juggling yourself

In this paper we clarify this proposition and the underlying principle "interaction creates meaning". Subsequently, we illustrate on the basis of five of our projects which kind of methods, tools, knowledge and skills can help designers become and act as experts in movement-based interaction. We start by explaining what we mean by movement-based interaction and why we consider this as one of the core topics of product design.

\section{What is movement-based interaction?}

Most products are tangible and require by nature that one holds them, moves them and operates functional parts. In our opinion, that is not the core of movementbased interaction, especially in the area of digital products. The essence lies in the meaning and richness of the movement as Djajadiningrat states:

"From a product design perspective, however, tangibility is not the most challenging concept within augmented reality. Clearly, unlike software, electronic consumer products are tangible to start with. What is new is not so much the tangibility of the interaction as the richness of the interaction." [1]

We clarify the concept of movement-based interaction with two design concepts: Puppy and Explorascope.
They give an impression of the scope of this design area.

\subsection{Puppy: an expressive music stress reliever}

Let us compare a traditional MP3 player with Puppy, a prototype of a MP3 player that is designed to reduce stress, see Fig. 2. One generally operates a regular MP3 player by pushing buttons or moving (touchsensitive) scroll wheels and sliders. The actions are rather standard and poor. Within the Interactive Technology Design course at the Delft University of Technology, we asked students to design an expressive music stress reliever that addresses specific human values, based on the human value theory from social psychologist Schwartz [2]. Every team got a different set of values, for example, benevolence, universalism and self-direction, or achievement and power. Puppy is designed for someone who considers benevolence and security as important personal values. The player exploits the expressive rich repertoire of movements and is operated by gentle and subtle gestures, where we define a gesture as a movement of one's body that conveys meaning to oneself or to a partner in communication, where that partner can be a human or a
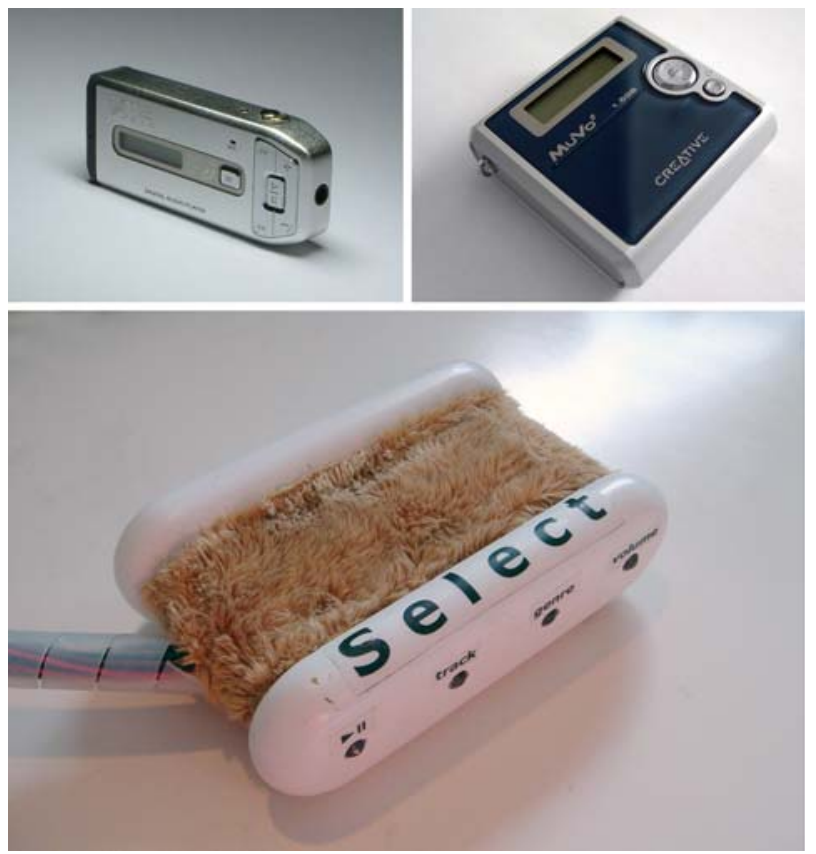

Fig. 2 Top Regular MP3 players are operated by buttons, (touch-sensitive) scroll wheels and sliders. Bottom Puppy, a MP3 player to reduce stress, is operated by stroking where the place, direction and character of the stroke determine how the functionality is processed and executed (designed by Master student Johannes Zachrisson for the course Interactive Technology Design at the Delft University of Technology) 
computer [3]. Stroking on the select side of Puppy allows one to switch between functions (play/pause, track, genre, volume) and stroking on the +/- side increases or decreases the chosen function. The direction of stroking and the character (speed, area of touching and the length of the stroke) determines how the functionality is processed and executed. A first experiment shows that people label Puppy indeed as designed for benevolence and security mainly due to the way of interaction.

\subsection{Explorascope: an interactive education toy for multi-handicapped children}

Explorascope is developed within LinguaBytes, a project, which is carried out by the Delft University of Technology, Viataal-Research, Development \& Support (RDS) and Radboud University Nijmegen. LinguaBytes aims for the development of an interactive educational toy in order to stimulate the language and communicative skills of multiple-handicapped children between 1.5 and 4 years old. Explorascope (E-scope) enables young children with severe physical and cognitive handicaps to learn simple concepts (e.g. sleep, clock, bear) through tangible interaction and play. In contrast to most of the programs that are currently available, we do not place these young children behind a screen with a special keyboard and mouse, but we give them a toy-like computer where they can play with. One can use E-scope on a table with an integrated horizontal screen or a separate monitor, or it can be used on the ground in combination with drawings (see Fig. 3). The upper ring can be turned (automatically with an addition input device if necessary) to zoom into stories or browse through menu-structures, several buttons can be pushes to select symbols that have to be learned and the entire E-scope can be moved and placed, e.g. above drawings to trigger interactive stories. E-scope can be adapted to an individual child with respect to interaction possibilities and the level of difficulty of the stories and concepts. The first test with children in the Sint Maartenskliniek shows that despite their physical limitations, both children and therapists are enthusiastic about the movement-based and playful character of the educational toy.

\section{The importance of movement-based interaction}

We believe it is important to design for movementbased interaction. Our motivation arises partly from the present range of digital appliances, for example the two upper MP3 players shown in Fig. 2. When we consider these digital appliances we see that the appearance of the devices and the interaction with them differs slightly. Nevertheless, the underlying principle of digital appliances is in most cases the same: they resemble PCs as for input (buttons), output (screen-based display) and structure (menus and decision trees). Although these little PCs are no longer a means to do our work but help us to pursue our lives [4], they seem to have problems separating themselves from their big brother.

Until recently, research on human-product interaction has concentrated on cognitive skills. Because intelligence has no form, design research, quite naturally, turned to the intelligent part of humans and thus to the science of cognition to find answers. This has resulted in interface design that places a heavy burden on human intellect [5]. Of course, many of us like their iPod, Creative Zen or Sony NWA3000 v and experience no problems while trying to access all functionalities. But people are different and our studies with personalised products, such as the one we did with Puppy, shows that people prefer products that are tuned to their personality [6, 7]. Not everyone prefers these little PCs. We regret the limited scope of digital products, where electronic circuits and microprocessors have broken the intrinsic link between functionality, appearance and actions, thus offering designers the oppurtunity to create a whole new perspective on design.

Moreover, finding new guiding principles for design is difficult, especially in our contemporary culture that lost its unifying ideology [8]. In response to the $\operatorname{cog}$ nitive-oriented uniformity of digital appliances, many designer-researchers advocate for embodiment and for capitalising on all human skills, including emotional and perceptual motor skills $[5,9,10]$.

We consider two reasons paramount in turning to embodied interaction. Firstly, this turn to embodied interaction can get human and social values back in balance. After the modernist tradition with its technology push and its passion for the logical, we now enter an era that shows respect for a person as a whole (with a mind, heart and body) and exploits all his skills [11]. Moreover, technology influences our culture and people's everyday lives, and embodiment can help to shape people's engagement with reality [12].

Secondly, movement-based interaction provides us with information about the world around us and ourselves. This basic principle, which is the leitmotiv for all our work, is rooted in Gibson's ecological theory of perception [13]. Gibson states that the world unfolds itself in possibilities for action. We perceive the world in terms of what we can do with it. It affords action to an organism on the scale of that organism. For 

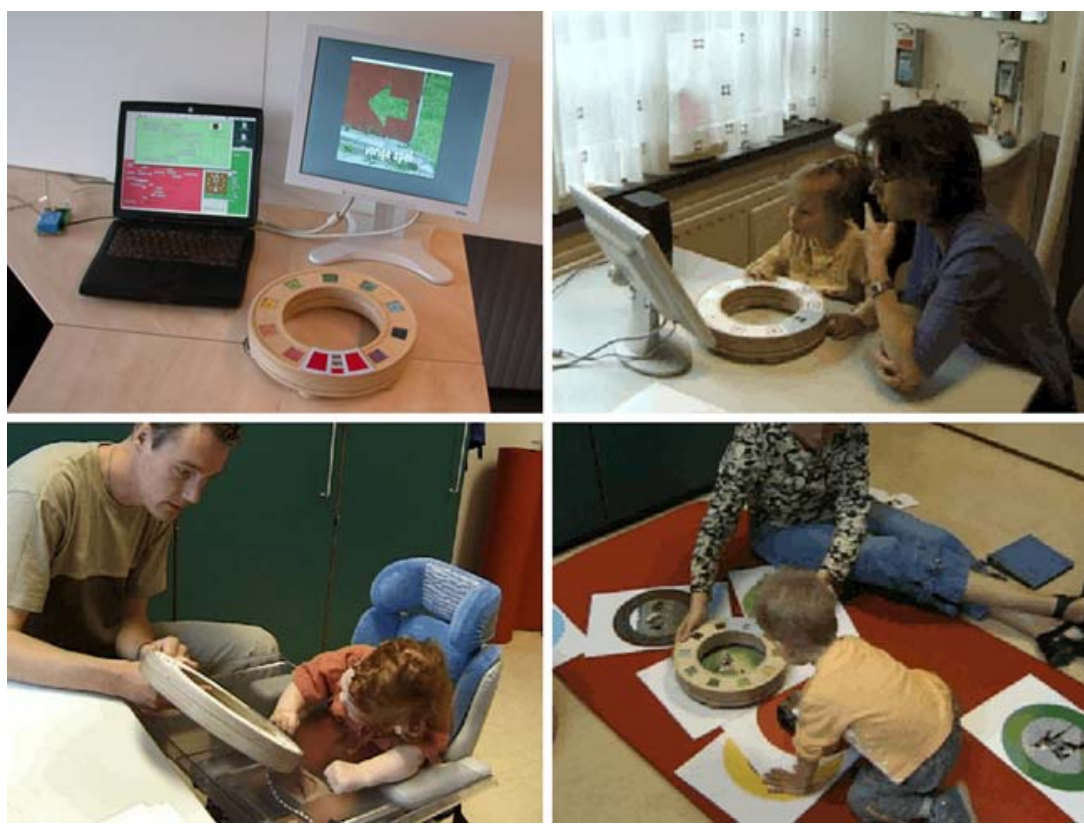

Fig. 3 Explorascope is an interactive educational toy for multihandicapped children. The complexity and possibilities of the stories, the interaction and the configuration can be adjusted to an individual child. For example, Child1 prefers moving the Escope over the table, turning the upper ring and pushing the Picture Communication Symbols $(P C S)$ on the wooden ring to

example, a chair affords sitting to an adult person, and it affords stability to a small child who wants to stand up. Thus, the world is inherently meaningful for our body and by moving we can get access to that meaning. Interacting with products creates meaning for a user.

Now let us assume that we agree upon the importance or opportunities of movement-based interaction. We postulate that if one truly likes to design for movementbased interaction, one has to be or become an expert in movement, not just theoretically, by imagination or on paper, but by doing and experiencing while designing. So, besides having knowledge of the salient aspects of embodied interaction, one has to move in order to design movements. Our argumentation is based once more on Gibson's ecological theory of perception. Because interaction creates meaning, it can stimulate designers to explore, study and design the relationships between a variety of aspects such as sensation, dynamic character, story, interaction style, experience, emotion, function, form and semantics. Especially when designing for rich interaction these relationships are too complex to trust solely on imagination or non-temporal visualisation and brainstorming techniques. Moreover, when externalising one's ideas through, e.g., models, one can also discover how potential users experience the concepts while using them. hear and see stories on a separate screen (top right). Child2 prefers having an E-scope with an integrated LCD display which can be operated by eye-movements (bottom left). Child 3 can be persuaded to listen to short stories by rolling the E-scope over large drawings that are lying on the floor (bottom right)

Therefore, we develop design tools that support this expressive, rich behaviour, and find techniques and methods how to design for movement-based interaction. We show on the basis of five of our projects which kind of methods, tools, knowledge, skills, etc. can help designers become and act as experts in movementbased interaction. For each project, we explain our basic assumptions, the design itself and the lessons we have learned from it. We conclude this paper with our general findings and recommendations.

\section{ISH: an interactive installation to generate knowledge through reflection-on-action}

When pursuing the next generation of digital products, especially intelligent and adaptive products, we have to find ways to study and measure the complex interdependent relationships between products, users and context. Research through design seems a useful approach that can address this kind of complexity. Through the act of designing resulting in experiential prototypes, and subsequently testing these prototypes preferably in real-life setting, scientific knowledge can be generated about human-product interaction, more specifically movement-based interaction. 
In this spirit, we have designed, built and tested a range of experiential products and installations over the last few years that enabled us to study the complexity of expressive tangible interaction [7, 14]. By actively exploring, manipulating and analysing the characteristics of these interactions, we try to find the salient aspects of expressive tangible interaction. We study four salient modes of interaction [14]:

1. Interaction mode. What style of interaction is used? What is the influence of physical and experiential aspects of the interaction such as interactivity (e.g. complexity, type of bodily movements, actionfeedback mapping), vividness (e.g. richness and abundance), engagement, flow, excitement, and expressivity of both product and behaviour [15, $16]$ ?

2. Activity mode. What is the character of the interaction? For example, is the interaction an experience-oriented activity or a goal-oriented activity [17] and does the type of interaction enhance usability and straightforward use and/or experience and exploration?

3. Human mode. Who is interacting with the product (characteristics, belief, human values, concerns, personality, skills, senses, effectivities,...) and what is the social dimension, e.g. are people working together or alone?

4. Context mode. Where, when, and how often, long, frequent, etc. is a product used?

Finding salient aspects of movement-based interaction can help designers to tune their products towards individuals, enhance embodiment and capitalise on all human skills.

\subsection{Design}

In order to get more knowledge about movement-based interaction, we started building ISH a few years ago [18]. ISH is a social and tangible interaction playground that allows us to evaluate various aspects of interaction through loops of (re)designing, building and testing. At the moment this dynamic research environment consists of a square rack with a horizontal projection screen in the middle and an evolving set of tangible devices, of which a selection can be placed on top of the rack, see Fig. 4. Every device has its own character with respect to, e.g. feedforward, feedback, time-delay, use of hands, intimacy, engagement, richness and clarity. Sensors in the devices are connected to a computer, which is positioned below the screen. The computer runs the ISH program, which is implemented using a software development environment based on real-time dataflow modelling (Max/MSP). This approach allows us to iterate quickly from idea to prototype. We can change the program while it is running to, for example, alter the mapping of input to function in and among the connected devices. In addition, it enables us to record user actions for analysis purposes.

We examine the activity mode by experimenting with three application domains: (1) playing a social interaction game (mainly experience-oriented), see Fig. 5, (2) visually searching and selecting images from a database (mainly goal-oriented) and (3) composing music and images (both goal- and activity-oriented).

Within these three application domains, the relation is studied between interaction mode (product characteristics and interaction style) and human mode (individuals and their social behaviour). In the near future we will also include the context mode.

\subsection{Lessons learned}

While developing and adjusting ISH over the last 5 years, we have experienced that reflection-in-action and on-action [19] can be stimulated through loops of designing, building and testing products with an experiential quality. This reflection on what is done by the designer and user during interaction constitutes design knowledge and helps us to generate new hypotheses, which hopefully enable us in the long run to formulate the salient aspects of not only movement-based interaction, but also resonant interaction [14]. We consider installations extremely useful as generators of conditional laws; laws which we test again by designing appliances based on these laws and reflecting on the actions and experiences of users when interacting with them, as we have done so far with MP3 players and expressive music stress relievers.

Up till now, we can draw several conclusions based on the explorative studies with ISH. Firstly, our observations and interviews show that not all types of tangible products and interaction lend themselves for expressive behaviour. An extremely simple device such as Little Knobbish, which consists of two small knobs that track the speed, direction and the relation between these two aspects, is hardly supportive when aiming for expressive behaviour. More rich gestural interfaces can allow and stimulate people to exploit the expressive rich repertoire of movements, which enable them to express their feelings. Striving for gestural interaction could open up interesting areas of human-product interaction.

Moreover, we have found that personality plays a role too. Not all persons prefer or are able to use such expressive tangible devices to the max. Although the feeling of sand through one's fingers made Big 


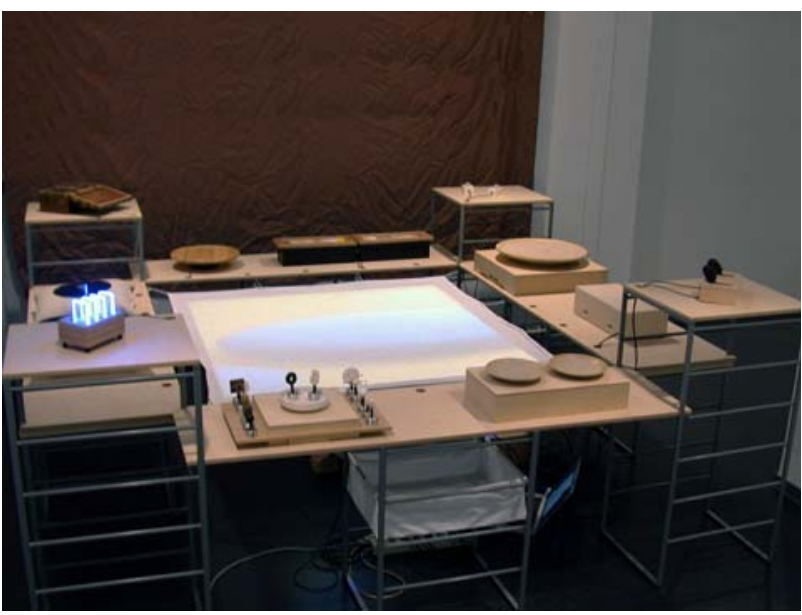

Fig. 4 The interactive installation ISH uses a variety of tangible objects to explore the relationship between interaction mode, activity and human mode

Grubbish very attractive (see Fig. 5 for further explanation), the necessity for exploration and a somewhat fuzzy relation between cause and effect was more

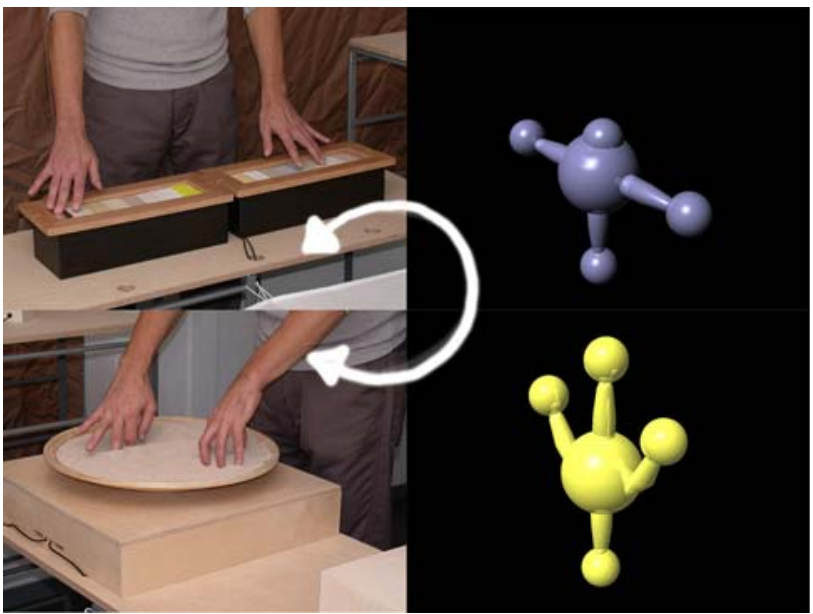

Fig. 5 The social interaction game called TOUCH stimulates social interaction. By expressing one's feelings through the tangible devices, one adjusts the behaviour and appearance of animated characters projected on the screen. These characters interact with and respond to each other, which in turn can invite players to adjust their actions. For example, we have two persons interacting simultaneously with ISH. One is operating Touchish, which consists of two big expressive touch sliders that have gradient texture patterns (from soft to rough and from fine to course). The position of the fingers are tracked with strain gauges and linked to the selection algorithm. The other person is operating Big Grubbish, where the position of the hands within a dish of sand, as well as the orientation and speed of the movements, are tracked with strain gauges and linked to the selection algorithm. If the first person is caressing Touchish with his fingers rather hesitantly (slow movements that exert low, slightly fluctuating pressure) a rather shy moving character is created and projected, which can be opened up by a friendly character that is in this case created by playfully moving through the sand of Big Grubbish. The game is not yet fully operational attractive to imaginative and experience-oriented persons than to goal-oriented persons. We have found similar results with other tangible products and installations $[6,7]$. The need for expressive movement-based interaction depends on the person, the problem at hand and the context of use.

Finally, the way people interact with ISH seems to correspond with general characteristics of two-handed interaction where both hands have their own specific function, as described by Guiard [20] and Buxton and his colleagues Kabbash and MacKenzie [21]. The nondominant hand offers a spatial referential framework for the movements of the dominant hand. Its actions precede the actions of the dominant hand. Finally, the dominant hand makes more refined movements with a higher temporal frequency. These characteristics can be capitalised on by designers and applied to challenge users to enhance their motor skills and pride when becoming experts in movements, e.g. when feeling enjoyment and pride while mastering an instrument.

\section{Choreography of interaction}

Design and dance have many things in common, which the Bauhaus School in Germany already recognised and promoted. They encouraged combining different disciplines. A key figure in the area of dance was Oskar Schlemmer (1888-1943) with, e.g., "Triadische Ballett", which can be seen as a forerunner of today's mixed media theatre [22].

Designers can benefit from knowledge and experience from the dance profession, now that the focus of design is shifting from the product itself towards interaction. We believe that choreography (including dance improvisation and movement analysis), which is essential for dance, is extremely valuable for design too. This belief has led to the Design Movement approach. The third author has been developing this approach for the last 4 years [23]. In this approach, design is focused on creating activities and movements, more specifically on a choreography of interaction. The product itself comes into existence in the choreography of interaction. It is felt and hence discovered as a trace and missing link; the link that is needed to fit and to complete the choreography of interaction. The next example will show how choreography can play a role in the design process and how it affects the design outcome.

\subsection{Design}

Instead of designing a vase directly, the third author took flower arranging as a starting point for this pro- 
ject. The final design of the vase is based on four principles that surfaced when experimenting with arranging flowers. First, the centre of gravity of the flower's weight instigated her to hold the stalk just under the corolla, resulting in an elegant, stable balance and caring lift. Second, the both careful and greedy admiration for flowers instigated her to slide them between her fingers, enabling her to hold more flowers and protectively lift them in the palm of her hand. Third, as a result of the greed of admiration she discovered a whirl of both arms, turning in relation to each other while bringing in and composing a plane of an increasing amount of flowers. While doing so she felt a fourth, not yet possible motivation, to embrace more flowers than she was able to. By actually arranging the flowers and deliberately choreographing the interaction, the final design 'Commit' quite naturally arose from the interaction with the flowers, as if it were a solidified reflection of her movements. Ideally, Commit should evoke these kind of movements and feelings again, when a person interacts with it (see Fig. 6).

\subsection{Lessons learned}

Choreography of interaction opens up a whole new range of interactions and products that have never been addressed by designers before. It truly places movements in the centre of the design process, and sees the product as a natural consequence of the preferred interaction. It is supreme embodied interaction that uses the full potential of designers and users, both

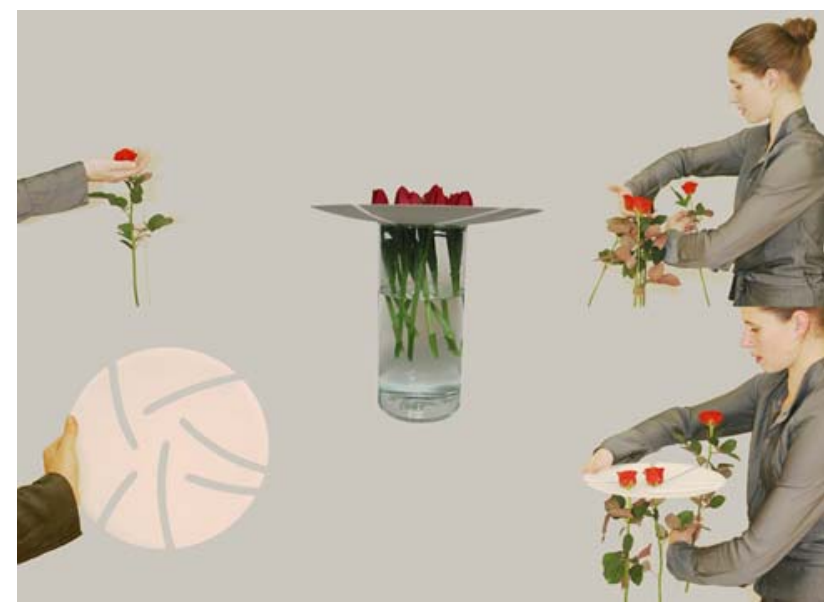

Fig. 6 Commit is a vase that has a circular shape and curved grooves that fit the whirl of two turning arms and hence the sliding inwards of flowers with a fluent rhythm, one after another. The slightly bent plane with grooves relates to the elegant lift of the growing weight of flowers, carried in the embraced personal space on a perceptual-motor as an emotional and experiential level.

When thinking about dynamic systems, one can also use this choreography to design the expressive actions and movements of the product itself, as Djajadiningrat and his colleagues [24] show with their dynamic emotion-expressing products. It is a challenge to explore and experiment how these products fit with its user actions.

Our experience with design students choreographing interaction indicates that designers are able to express themselves this way. Even more, they like working this way and see the potential of the approach. Nevertheless, to get beautiful choreographies of interaction, we have to educate design students to trust their bodies, to dare being expressive and teach them the principles of choreography and dance [23, 25]. Notwithstanding the aspect of familiarisation and training, interaction of choreography might not be applicable for all designers and all design problems. Therefore, it should not replace the existing design methods and tools but complement them.

\section{Expressive power of gestures}

Choreography of interaction calls upon the imaginative qualities of the designer to see the product as a frozen reflection of his movements. Up till now, we do not have any tools to visualise these reflections, let alone manipulate these visualisations. Tinkering material or props help to bridge this gap, as we have noticed during workshops and courses on interactive tangible sketching (see also Sect. 8). However, one can also try to use advanced technology to form the bridge.

Since 1993, the first author explored the potential of digital gestural design tools that support the designer in creating expressive tangible interaction concepts. [25]. In this section we will discuss the adequacy of gestures to capture and visualise geometry and expressivity during the design process. In the Sect. 7 we show one of the gestural design tools that was developed, called Attending Theremin.

\subsection{Studying gestural design}

Gestures are extremely suitable for a new design tool, because they can convey emotions and expression, as well as geometry and (inter)actions. Moreover, they can stimulate the creative process. Firstly, because socalled pathic gestures are made to support the thinking process, i.e. gestures with no intrinsic meaning towards form are used to maintain discourse. Secondly, gestures 
can be indeterminate and ambiguous, which stimulate imagery and consequently idea generation [26].

The first author performed three studies to make a first step in finding rules and patterns with respect to the meaning of gestures in order to implement a fullfreedom gestural design tool.

The first investigation was conducted to explore the appropriateness of gestures, and the working methods and strategies used when visualising a product with gestures, in this case a mineral water bottle. The study centred on conveying geometry and (inter)actions. Although we asked the subjects to visualise the bottle using gestures, the subjects did not receive any visual feedback during the sessions. They had to rely on their imagery.

The second investigation was performed to test a preliminary full-freedom gestural sketching system using a Wizard of $\mathrm{Oz}$ approach. In this case, the first author impersonated the computer. The subjects visualised with gestures several products provided on photographs, the Wizard interpreted their gestures and provided them with almost real-time visual feedback on a monitor, see Fig. 7. The subjects stopped the session when they were satisfied with the picture on the monitor as compared to the photograph.

A third study was conducted to test the premise for that the expressivity of gestures is subtle enough and even surpasses that of traditional sketching when capturing expressive design concepts. Moreover, the premise was tested for that outsiders are able to pick up the expression of gestures. If people cannot detect the expression, it might be hard for computers to do so.

Scents were used to make the expression of gestures measurable. The study encompassed a design part and two matching experiments, of which we will describe the second one. During the design sessions, design and dance students made four dynamic sculptures expressing the scents. The dance students expressed the sculptures in dances. The design students made half of the sculptures through traditional sketching and half through gesturing. After every gestural session, the designers sketched their virtual design on paper. At the end of the design part, the students evaluated all designs with respect to satisfaction and the two methods used with respect to appropriateness for design. Moreover, an independent interpreter visualised her impression of the dances and gestures (shown on videotape) in a sketch. This resulted in six types of visualisation as is shown in Fig. 8.

In the second matching experiment, subjects matched the interpreter's drawings of the designers gesturing (bottom middle Fig. 8) with the sketches of the virtual design created by the designers themselves (centre Fig. 8). Furthermore, they matched the films of a dancer (top right Fig. 8)with the sketches made by the interpreter when watching the dancers performing (bottom right Fig. 8).

\subsection{Lessons learned}

The results of all three studies show that there are huge differences between individuals in terms of design approach, gestures used and the use of both hands. But this does hardly influence the legibility and detection of the meaning of gestures. In general one can say that it is possible to convey and detect the meaning of gestures, with respect to geometry as Fig. 9 shows, as well as expressivity as was found in the last study.

When comparing existing tools like sketching with gesturing, the third study shows that there is no significant difference between the satisfaction of designers in respect to the sculptures made with sketches or with gestures. Although sketching is considered more suitable, gesturing is still believed to be an adequate design tool. These results are promising especially when considering the fact that design students have extensive training in drawing and none in gesturing.

The results from the matching experiment show that the interpreter was significantly able to capture the expression as intended by the designer or dancer, although the percentage of correct matches is lower for dances-drawing matches in comparison to drawing-drawing matches. It appeared that not only did the interpreter have to capture
Fig. 7 The Wizard of $\mathrm{Oz}$ could see the design student on her monitor by means of two cameras. The design student received visual feedback on a monitor. After the sessions, all the sketches were placed in a storyboard. On the right 16 out of 40 shots from one of the sessions in which a design student visualised a toilet
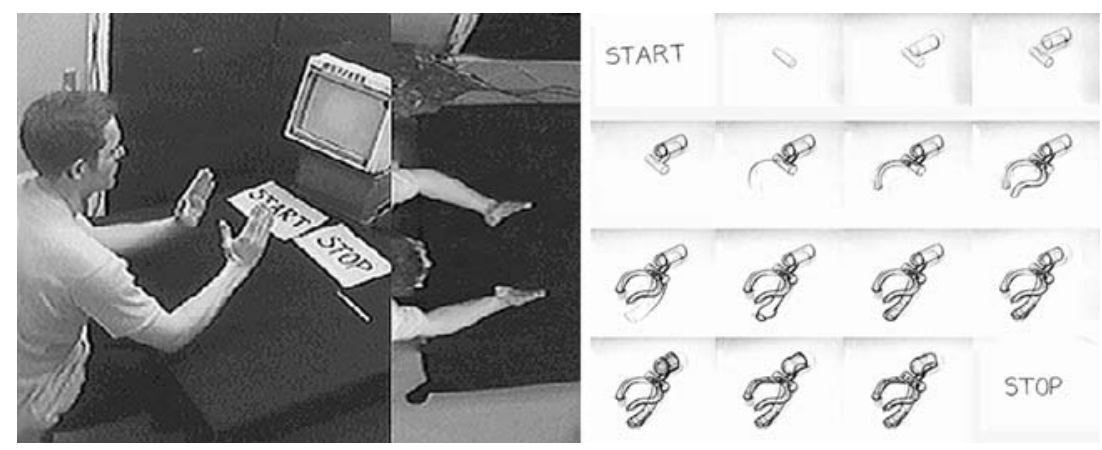
Fig. 8 Six different types of stimuli were created and matched to study the expressive power of gestures. Top Design and dance students created a sculpture or a dance. Middle Design students visualised their design made with gestures in a sketch immediately after they finished the design. Bottom An interpreter visualised the gestures and dances in a sketch, when seeing the video recordings of the design and dance students

Fig. 9 The Wizard sketched her impressions of the three products which were unknown to her, immediately after the session of the last subject in the second study. These sketches show a high resemblance with the original photographs
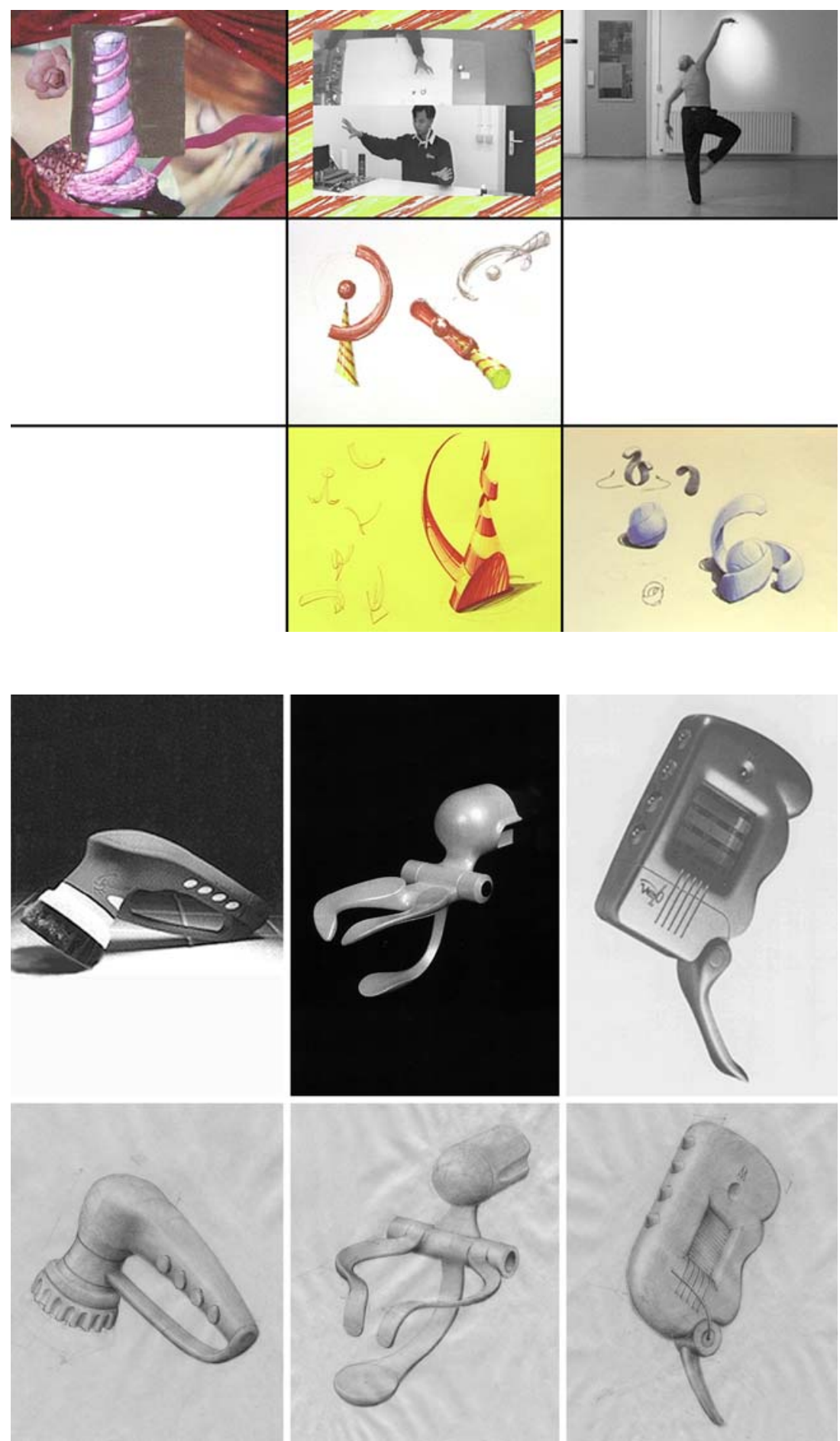

the expression correctly, the subjects performing the matching experiment also had to have the affinity to detect the expression. In conclusion one can say that both geometry and expressivity is conveyed by gestures, which is beautifully exemplified in Fig. 10.

Finally, knowledge about the expressivity of gestures can be useful for developing design tools, but it is at least as important for designing the choreography of interac- tion and the resulting product itself. Therefore, we should teach design students the expressive qualities of gestures and the principles of aesthetic interaction too, when educating them the basic principles of form-giving such as form-integration, abstraction, balance, rhythm, proportion, expression, colour, size, material, texture, tactility and sound. Details about these gestural studies can be found in the first author's doctoral thesis [25]. 
Fig. 10 The dance in which Sjoukje Philips expresses Ligustral (top left) is captured by Roselien Steur (the interpreter) in the sketch shown top right. Both movement and sketch resemble the gestures and sketch made by Wing-Ken Cheung (middle left and middle right). The sketch Roselien made of the gestural expression of Wing-Ken is shown on the bottom right. All three drawings of sculptures are highly similar with respect to shape and colour. This is all the more striking, because the dance apparently expressed a greenish colour
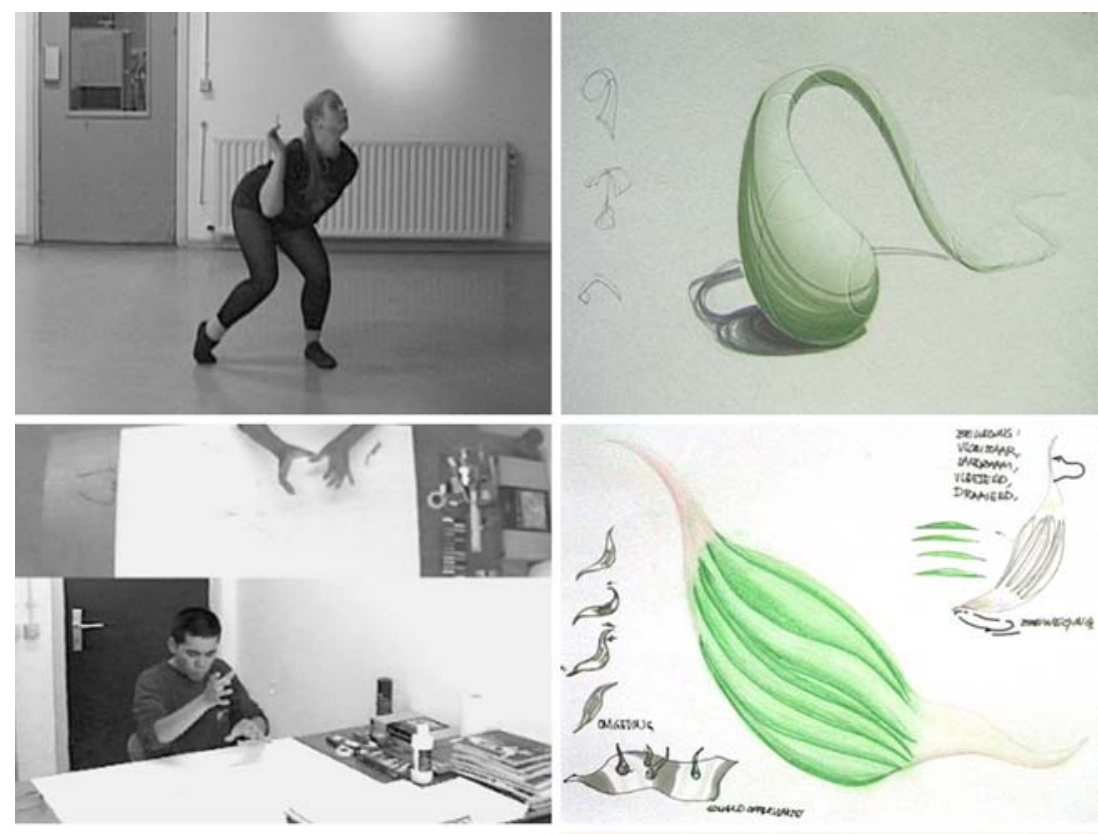

\section{The Attending Theremin}

Although some designers have a background in dance like the third author, most designers are not trained to use the expressivity of their body to explore interactions. The previous example shows that designers are still able and willing to express themselves this way. Nonetheless, visualising is a skill and if designers are not able to visualise their ideas it can limit their expressive scope.

Can a tool empower designers to browse through and adjust design solutions offered by the computer based on the expression of his gestures, without having to worry about structural aspects such as geometry?

\subsection{Design}

The Attending Theremin is derived from a musical instrument called the Theremin, which is played by changing the distance between the hands and two antennas which measure the body capacitance, i.e. the amount of body (influenced by size and distance) that is situated near the antennas. The design version basically works the same, although it changes designrelated aspects instead of music. The Attending Theremin has a set of antennas, all with their own characteristics, to adjust e.g. shape, colour, texture, material, size, sound and movements of a product [25]. Moving the hands next to the different antennas can change the $n$ dimensions of a product simultaneously or separately. The intensity of capacity (proximity of the hand and the antenna in relation to the volume of the hand) and the character of these movements (speed, flow and fluctuation) determine how the object changes its expressivity, as is the case with ISH products like Touchish and Big Grubbish, see Fig. 11.

The Attending Theremin was envisioned to work with a desktop VR system on the basis of the space through (head) movement principle, but could also make use of state-of-the-art displays. We have built several preliminary versions, with different shaped antennas and different variations in characteristics (see Fig. 12). 


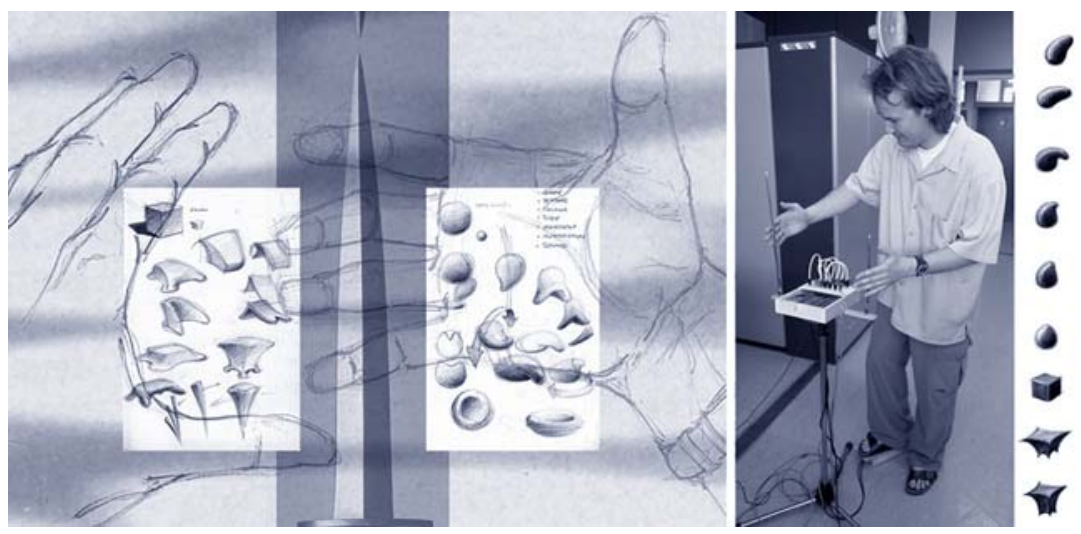

Fig. 11 Right A preliminary working version of the Attending Theremin. The subject is moving his hands around two antennas. The body capacitance that is measured by the left antenna influences the orientation of a simple 3D shape that is shown on a monitor. The capacitance measured by the right antenna is used to alter the expression of the object from, e.g., shy to aggressive (see sequence of nine shapes on the right of this

\subsection{Lessons learned}

The Attending Theremin works as an instrument that invites and seduces the designer to explore and play it. Although the antennas merely detect capacitance, the designer can exploit the aesthetics of his own movements. He is free to make any movements he likes or sees fit to change the product and the interaction, which was confirmed by the observations we made. All designers had a personal style of using the Attending Theremin, from reserved to exuberant, from concentrated working to a superficial flirt and from finger fiddling to full body movements. Additionally, most of them felt a state of flow while operating and found it
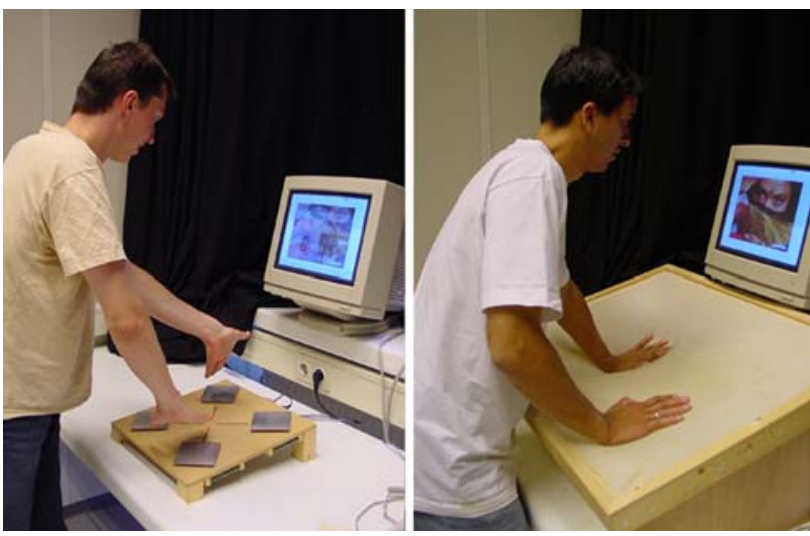

Fig. 12 Different forms and number of antennas were studied, as well as the use of tactile and force feedback. In this case the character of the movements and the intensity of the capacitance enables these designers to adjust the expression of the image (changing shapes, composition and colour). figure). The expression is enhanced by expressive audio feedback (the actual sound of the original Theremin). The dynamic coupling of user actions and visualisation makes it possible to animate the shape real-time. Left Collage of the Attending Theremin, indicating that one can browse through a variety of images using one's hands

sometimes hard to stop, especially with the first version shown in Fig. 11.

Designers see the computational power as potentially useful, as long as it stimulates their creativity and leaves them in control. It is an extremely quick way to evaluate alternatives, although it needs extensive development in order to produce interesting movement-based design concepts.

Finally, the playful character of the tool can tempt designers to explore their expressive movement vocabulary. Gesturing without feedback sometimes feels awkward for designers, especially when they are not trained in movements and dance. Here feedback is given.

\section{Interactive tangible sketching}

The last technique we discuss also makes extensive use of technology, but in a quick and dirty way.

Most design methods and tools for the conceptual design phase have problems addressing the dynamic aspects during interaction, especially when the integration of the physical and virtual is required. Now that products are becoming adaptive and intelligent, the tools are becoming even less suitable. Moreover, the speed of making and testing a 3D sketch is sometimes problematic, especially in combination with the experiential character of this tangible sketch.

The last few years, Aadjan van der Helm together with the first author tried to integrate several tools and techniques to enable interactive tangible sketching. Key aspects of this technique are the focus on 
rich tangible interaction, the speed of making and testing a 3D sketch, and the experiential and interactive character of the sketch. Moreover, the technique should enable the designer to focus on the action potential of physical objects as generators of meaning in interaction.

\subsection{Tool}

Interactive prototyping integrates physical modelling, more specifically tinkering, with play-acting, sensor technology and dataflow modelling programs.

Spatial models have the advantage that they can be seized, turned, moved and used, which make them appropriate for exploring movement-based interaction. Interactive tangible sketching combines tinkering, i.e. a brainstorming technique that produces fairly lowfidelity, prototypes with a high-interaction relevance [27] with sensor technology and the dataflow modelling program MAX/MSP. We use Phidget Interfacekits, including an extensive set of (plug-and-play) sensors and actuators to incorporate in the models. The kits are connected to a computer using a software development environment based on real-time dataflow modelling (Max/MSP) as is shown in Fig. 13. This approach allows designers to iterate quickly from idea to prototype. They can change the program while it is running. Moreover, we refined our set of tinkering materials, from rather low-profile hobby materials (straws, elastic bands, balloons, etc.), to more refined and sophisticated parts and materials. This way the designers are stimulated to focus their attention on the expressive richness of interaction, including subtlety.

\subsection{Lessons learned}

We have conducted several workshops and courses at, e.g., the DPPI05 conference at the Technical University Eindhoven, Microsoft Research Cambridge and the Interactive Technology Design course at the Delft University of Technology, which were used to letting the participants experience the possibilities of interactive tangible sketching.

The results show that participants are able to build an interactive tangible sketch within a few hours or even less (depending on the complexity of the design). These resulting 3D sketches were sufficiently convincing to be tested on an experiential level with users.

The method stimulates to design products which place tangible interaction in the centre, as the designs show. Most participants were able to design and evoke expressive movement-based interaction, which was enhanced by diversity and expressivity of the materials offered. In general, the technique is considered being fun, stimulating and satisfying to work within this interactive dynamic way.

Through several design loops, the ideas can quickly be developed further (both conceptually as well as physically) which appeared to enhance the richness of the interaction. During this process the models slowly converge towards interactive tangible prototypes with more refinement, certainty and decisions made.

\section{Conclusions and future work}

In this paper we advocate movement-based interaction, because the world is inherently meaningful for our body and by moving and interacting we access this meaning. This principle holds not only for users but also for designers when generating ideas and developing concepts. Therefore, we postulate that if one truly likes to design for movement-based interaction, one has to be or become an expert in movement, not just theoretically, by imagination or on paper, but by doing and experiencing while designing.

In order to do so, we believe that designers need design tools, techniques, knowledge, awareness and skills that support their search for expressive, rich behaviour. Based on our work in this, of which a few examples have been discussed in the paper, we would like to conclude with seven guiding principles we consider vital for movement-based interaction. The foundations for this principle can be found in the section(s) given in brackets.

1. Meaning through interaction. The world unfolds itself in possibilities for action, and we perceive the world in terms of what we can do with it. It affords action to an organism on the scale of that organism. Thus, the world is inherently meaningful for our body and by moving we can get access to that meaning. Interacting with products creates meaning for a user (Sect. 2).

2. Richness of interaction. Electronic consumer products are tangible to start with, so one should not focus on the tangibility of the interaction but on the richness of the interaction. Exploiting the expressive rich repertoire of movements, could challenge users to enhance their motor skills and pride when becoming a behavioural expert in a certain domain. But it could also stimulate their feelings, not only by feeling pride that they have mastered a certain skill, but also by enabling people to express their feeling through movement. (Sects. 1, 3) 


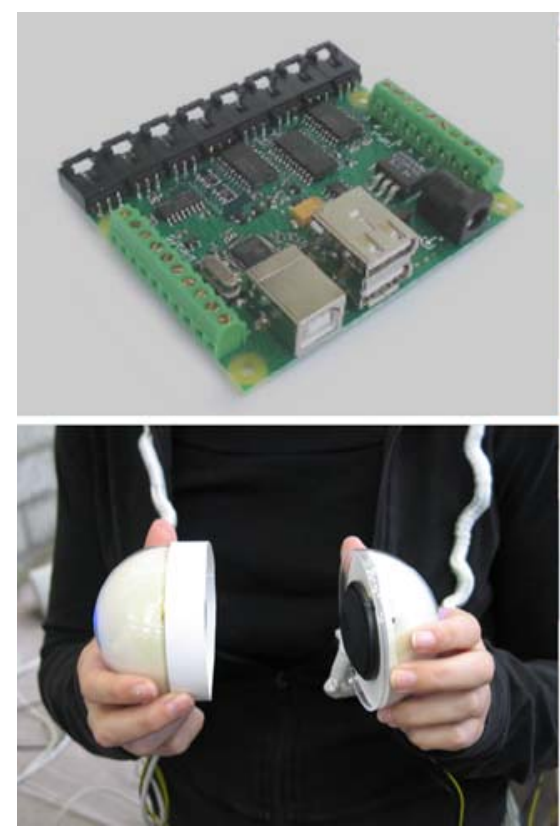

Fig. 13 Top left Phidget Interfacekit. Top right The dataflowmodelling program MAX/MSP is used to control the product's behaviour in combination with the sensors and actuators. Bottom left "Matchmaker" is designed for social interaction. Pushing the two halves of the sphere against each other and turning them in opposite direction selects a different mood (different music genres and changing lights in the sphere) The volume is dependent on the mode. In private mode one person holds the shells against his ears, and the volume is turned low. In public

3. Design by moving. Now that the focus of design is shifting from the product itself towards interaction, we need new methods and techniques to design these interactions and accompanying products. We emphasise the strength of exploring, discovering and creating through interaction, because interaction creates meaning (Sects. 1, 2).

4. Support for movement. Especially when designing for rich, tangible interaction the relationships between aspects such as dynamic character, interaction style, experience, function and form are too complex to trust solely on imagination or nontemporal and non-dynamic visualisation and brainstorming techniques. Designers need tools that help them to explore and visualise interactions, such as the Design Movement approach with its choreography of interaction, gestural design tools, installations and interactive tangible sketching. These tools should exploit the expressive rich repertoire of designers' movement to incite the expressive rich repertoire of the users' movements and even the product's movements (entire paper).

5. Research by doing. The strength and beauty of research through design lies within the possibility to address the complexity of all interlinked aspects

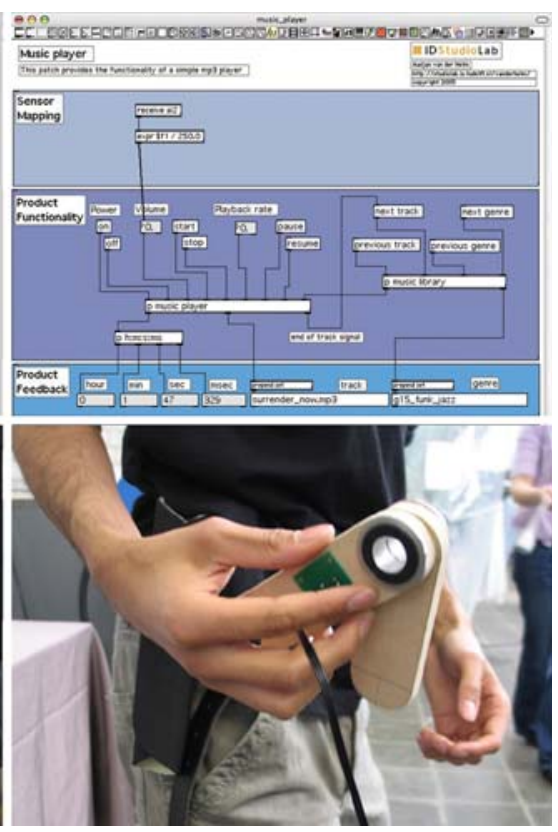

mode, the player is shared (two persons holding it against their ears) and the volume is increased. Bottom right 'Feeling Lucky Punk' could hardly have been designed and experienced with traditional design tools. By turning one of the pointers quickly around several times, one can change the genre of music. By turning it once, one can alter a song within a genre. These 'fully' working interactive tangible sketches were produced within a few hours

such as users, interactions, design, context and technology. It can address the richness and expressivity of the designer's and user's movements and experience, which is hard to do with traditional approaches. Moreover is enhances reflection-inaction and on-action again for designers and users, which not only constitutes design knowledge but also helps to generate new hypotheses (Sect. 3).

6. Educate through and for movement. If we want to shift the focus of design, we also have to educate our future designers. We have to provide knowledge and offer them methods, tools and techniques (including their own bodies) to facilitate the design process. We have to educate their senses so they become sensitive to richness, expressivity, subtlety, diversity and similar aspects (Sects. 4-7).

7. Design for diversity. The inherent meaningfulness of movements unfolds a world with possibilities for action, in accordance with the action possibilities of each person. Because we all have our own physical, emotional and rational characteristics, our own history in life and our own needs, our preferred movements will differ, and consequently we prefer different possibilities for action (Sects. 1, $3,5,6)$. 
Acknowledgments We like to thank Aadjan van der Helm, Rob Luxen, Gerda Smets, Tom Djajadiningrat, Stephan Wensveen, Philip Ross, Joep Frens, Hans van Balkom, Riny Voort, Jan de Moor, Pieter Jan Stappers, Rudolf Wormgoor, David Keyson, Paul Hekkert, Han Chul Jung, Johannes Zachrisson, Miguel Bruns Alonso, the Maeterlingschool in Delft and the Maartenskliniek in Nijmegen, as well as all participating students and colleagues who made these projects a success.

\section{References}

1. Djajadiningrat JP, Overbeeke CJ, Wensveen SAG (2000) Augmenting fun and beauty: a pamphlet. In: Mackay WE (ed) Proceedings of DARE'2000. Helsingor, pp 131-134

2. Schwartz SH (1992) Universals in the content and structure of values: theoretical advances and empirical tests in 20 countries. Adv Exp Soc Psychol 25:1-65

3. Hummels C, Stappers PJ (1998) Meaningful gestures for human computer interaction: beyond hand postures. In: Proceedings for the 3rd IEEE international conference on automatic face and gesture recognition (FG'98), Nara, Japan, 14-16 April 1998. IEEE Computer Society Press, Los Alamitos, pp 591-596

4. Gaver B (2002) Designing for Homo Ludens. I3 Magazine, vol 12, pp 2-6

5. Overbeeke CJ, Djajadiningrat JP, Hummels CCM, Wensveen SAG (2002) Beauty in usability: forget about ease of use! In: Green WS, Jordan PW (eds) Pleasure with products: beyond usability. Taylor \& Francis, London, pp 9-18

6. van der Heijden A, Hennink A, Kistemaker N, Hummels C (2005) Especially made for you. In: Wensveen S, Diederiks E, Djajadiningrat T, Guenand A, Klooster S, Stienstra M, Vink P, Overbeeke K (eds) Proceedings of the conference designing pleasurable products and interfaces, Eindhoven, The Netherlands, 24-27 October 2005, pp 478-479

7. Hummels C, Ross P, Overbeeke CJ (2003) In search of resonant human computer interaction: building and testing aesthetic installations. In: Rauterberg M, Menozzi M, Wesson J (eds) Interact '03. IOS Press, Amsterdam, pp 399-406

8. Branzi A (1989) We are the primitives. In: Margolin V (ed) Design discourse: history theory criticism. University of Chicago Press, Chicago

9. Dourish P (2001) Where the action is: the foundations of embodied interaction. MIT Press, Cambridge, MA

10. Fels S (2000) Intimacy and embodiment: implications for art and technology. In: Proceedings of the 2000 ACM workshops on multimedia, pp 13-16

11. Wensveen S, Overbeeke CJ, Djajadiningrat T, Kyffin S (2004) Freedom of fun, freedom of interaction. Interactions 11:59-61

12. Verbeek P (2002) Devices of engagement: on Borgmann's philosophy of information and technology. Techné J Soc Philos Technol 7(1):69-92
13. Gibson J.J (1986) The ecological approach to visual perception. Lawrence Erlbaum, London (Original work published in 1979)

14. Hummels CCM (2005) 'Descendants' of a design quest for diversity. Keynote speech. In: Wensveen S, Diederiks E, Djajadiningrat T, Guenand A, Klooster S, Stienstra M, Vink $\mathrm{P}$, Overbeeke $\mathrm{K}$ (eds) Proceedings of the conference designing pleasurable products and interfaces, Eindhoven, The Netherlands, 24-27 October 2005, pp 10-23

15. Wensveen SAG, Djajadiningrat JP, Overbeeke CJ (2004) Interaction Frogger: a design framework to couple action and function. In: Proceedings of the 2004 conference on designing interactive systems: processes, practices, methods, and techniques, Cambridge, MA, USA, pp 177-184

16. Frens JW (2006) A rich user interface for a digital camera. Pers Ubiquit Comput 10(2-3):177-180

17. Hassenzahl M, Seifert K, Pastoor S (2002) The effect of "Usage Modes" on product appeal. Available at http:// www.tu-darmstadt.de/fb/fb3/psy/soz/forvero_mh.html (Last visited 20-06-2006)

18. Hummels C, van der Helm A (2004) ISH and the search for resonant tangible interaction. Pers Ubiquit Compu 8:385-388

19. Schön DA (1983) The reflective practitioner; how professionals think in action. Basic Books, Jackson

20. Guiard Y (1987) Asymmetric division of labor in Human Skilled Bi-manual action: the kinematic chain as a model. J Motor Behav 19(4):486-517

21. Kabbash P, MacKenzie IS, Buxton W (1993) Human performance using computer input-devices in the preferred and non-preferred hands. In: Proceedings of InterCHI '93, pp $4747-481$

22. Anderson J (1985) In: The New York Times. Available at http://www.sitemaker.umich.edu/dancetech (Last visited 20-06-2006)

23. Klooster S, Overbeeke CJ (2005) Designing products as an integral part of choreography of interaction: the product's form as an integral part of movement. In: Design and semantics of form and movement. Proceedings of the 1st European workshop on design and semantics of form and movement. New Castle, UK, pp 23-35

24. Djajadiningrat T, Matthews B, Stienstra M (2005). Easy doesn't do it: skill and expression in tangible aesthetics. Pers Ubiquit Comput, special issue on movement-based design (this issue)

25. Hummels CCM (2000) Gestural design tools: prototypes, experiments and scenarios. Unpublished doctoral dissertation, Delft University of Technology, Delft, The Netherlands

26. Fish J, Scrivener S (1990) Amplifying the mind's eye: sketching and visual recognition. Leonardo 23(1):117-126

27. Hummels CCM (2004) Educating interaction, experience and diversity. In: Loyd P, Roozenburg N, McMahon C, Brodhurst L (eds) The changing face of design education. Proceedings of the 2nd international engineering and product design education conference, 2-3 September 2004, Delft, The Netherlands, pp. 247-254 